\title{
Energy Efficient Routing Protocols for Wireless Sensor Networks: A Survey
}

\author{
Komal Mehta \\ Student, Dept. of CSE, \\ Jaypee Institute of Information Technology (JIIT), \\ Noida, India.
}

\begin{abstract}
Wireless sensor network (WSN) is the prominent way as one of the auspicious domain. It is collection of motes that are equipped with wireless interfaces, small scalable having sensing capabilities. These sensors have restricted processing and computing resources. The main concern of this research work is to provide a brief idea of recent proposed algorithms by applying optimization technique using genetic algorithm is described after contemplating on their communication architecture, various applications, challenges, routing protocols and clustering particularly designed for efficient energy consumption and frame stable network.
\end{abstract}

\section{Keywords}

Genetic algorithm; energy efficient clustering protocols; throughput; lifespan; routing protocols

\section{INTRODUCTION}

Wireless sensor networks have extended through-out the world in recent years. It is defined as network of tiny devices called smart sensors or motes. These smart sensor nodes are devices provisioned with processor, analog-digital convertor, radio interface, memory, power supply, sensors. They are economical in contrast of traditional sensors [1].

$$
\left.\sum \text { (Sensing, Radio, CPU , Power }\right)=\text { Sensor }
$$

The processors dispense the mote management functions and perform data processing. It manages procedure that make sensor node collaborate with other node to carry out assigned sensing task. The analog-digital convertor is the interface between two devices for better deliberate accuracy. A radio interface is implemented for wireless communication to relay the data to base station. Memory is used to store the information executed by processor (i.e program) and data (which can be raw or processed).The rechargeable batteries are used as primary power source. Solar panels may be added to the node is used as the secondary power supply that harvests power from the environment .Sensor attached to motes are capable of sensing the temperature , humidity, light etc. It is distributed and work cooperatively to communicate information gathered by different nodes from the environment and transmits the sensed data to sink and which either uses data locally or is connected to other network. All sensor nodes have limited power supply and potential of information sensing, data processing. As the sensors are small, it is feasible to fit them into a smaller volume with more power and with less production costs. Sensors have ability to operate unattended in harsh environments to sense and periodically transmit data to the sink or base station. A variety of sensors may be attached to the sensor node to measure properties of the environment. According to their distinct characteristic, each application has its own design concept and implementation to suit specific requirements. In the past, sensors are coupled with wired

\author{
Raju Pal \\ Assistant Professor, Dept. of C.S.E, \\ Jaypee Institute of Information Technology (JIIT), \\ Noida, India.
}

lines. Today, this environment is integrated with the ad- hoc networking technology to promote inter-sensor communication [1].

WSN typically has little or no infrastructure. It consist of few tens to thousands sensor nodes working together to observe a region to obtain data about the environment. There are two types of WSNs: structured and unstructured. Unstructured WSN is one that contains impenetrable collection of sensor nodes. Sensor nodes may be deployed in an ad hoc manner into the field causes the network is left unattended to perform monitoring and reporting functions. The network maintenance such as oversee the connectivity and detecting failures is difficult in unstructured WSN since there are so many nodes deployed randomly. In a structured WSN, all or some of the sensor nodes are deployed in a pre-planned manner i.e. fewer nodes can be deployed at specific location to provide coverage with lower network maintenance and management cost [1].

There are various types of WSNs [2] focusing on different manner of node deployment as shown in table 1 . These nodes have some characteristics, objectives and some challenges as shown in table 2 .

Table 1: Types of WSN

\begin{tabular}{|c|c|}
\hline $\begin{array}{l}\text { Terrestrial } \\
\text { WSN }\end{array}$ & $\begin{array}{l}\text { Nodes deployed in pre-planned or ad-hoc } \\
\text { manner. } \\
\text { As battery power is limited, Solar cells are } \\
\text { used }\end{array}$ \\
\hline $\begin{array}{l}\text { Underground } \\
\text { WSN }\end{array}$ & $\begin{array}{l}\text { Nodes are deployed underground to } \\
\text { determine the underground condition. } \\
\text { As energy is main issue, additional sink is } \\
\text { required above ground. }\end{array}$ \\
\hline $\begin{array}{l}\text { Underwater } \\
\text { WSN }\end{array}$ & $\begin{array}{l}\text { Nodes are deployed underwater. } \\
\text { Acoustic waves are used for } \\
\text { communication. }\end{array}$ \\
\hline $\begin{array}{l}\text { Multimedia } \\
\text { WSN }\end{array}$ & $\begin{array}{l}\text { Nodes equipped with cameras and } \\
\text { microphones are deployed in pre-planned } \\
\text { manner. } \\
\text { High bandwidth demand, high energy } \\
\text { consumption, quality of service etc. are the } \\
\text { challenges. }\end{array}$ \\
\hline Mobile WSN & $\begin{array}{l}\text { Nodes that can move on their own and } \\
\text { having the ability to reposition and } \\
\text { organize itself in network. } \\
\text { When the nodes are within range of each } \\
\text { other than communication has done. }\end{array}$ \\
\hline
\end{tabular}

There is various application of sensor in most of the areas such as environmental monitoring, health monitoring, seismic and structural monitoring, tracking objects, tracking vehicles, human tracking, traffic tracking etc. 
To integrate multiple sensor nodes on WSN Platform each having different processors, radio components and storage, is most challenging as well as processing raw data is also issue as resource constraints. To support these sensor platforms, system software must be designed by J. Yick et.al in 2008 [1]

To enhance the overall performance of network, the management and control services are developed that take care of resource usage, task distribution etc. Some main concerns of these are [1]:

Table 2: Various parameters concerning WSN

\begin{tabular}{|l|l|l|}
\hline Characteristics & Objectives & Challenges \\
\hline $\begin{array}{l}\text { Dense sensor node } \\
\text { deployment }\end{array}$ & $\begin{array}{l}\text { Small size \& } \\
\text { low cost }\end{array}$ & $\begin{array}{l}\text { Limited energy } \\
\text { capacity }\end{array}$ \\
\hline Battery-powered & $\begin{array}{l}\text { Low power } \\
\text { consumption }\end{array}$ & Sensor location \\
\hline Storage constraints & $\begin{array}{l}\text { Scalability \& } \\
\text { Reliability }\end{array}$ & $\begin{array}{l}\text { Limited hardware } \\
\text { resources }\end{array}$ \\
\hline Data redundancy & $\begin{array}{l}\text { Self- } \\
\text { configurability }\end{array}$ & $\begin{array}{l}\text { Random } \\
\text { deployment }\end{array}$ \\
\hline $\begin{array}{l}\text { Application } \\
\text { specific }\end{array}$ & Fault tolerance & Data aggregation \\
\hline $\begin{array}{l}\text { Frequent topology } \\
\text { change }\end{array}$ & Security & Scalability \\
\hline
\end{tabular}

a) Localization: Process by a sensor node tries to discover its own location after deployment. Some techniques to find location are Beacon node, (who knows their position act as reference for the other nodes), Proximity-based (it uses the neighbour node to determine their position and then act as beacon for others).

b) Synchronization: It mainly concern with routing and energy conservation. To increase the network lifetime, data must be transmitted in scheduled manner that is less collision and retransmission conserved power.

c) Coverage: It deals with the deployment of nodes (preplanned or ad-hoc so that the monitored region is covered with high extent of reliability.

d) Compression and Aggregation: It reduces the communication cost and increase data transfer reliability. They are required when large amount of data is transmitted

- Data-compression: it squeeze the data size before transmission and decompress at base station .in this the whole information is retained.

- Data aggregation: data is collected from multiple sensors nodes and merge together to transmit to the base station. The individual information is not retained [1].

Keeping all these things in mind the objective of our research in WSNs is to meet the curb by initiate new design concepts, creating or enhancing the existing protocols and developing new algorithms.

Our survey is disparate from others as focused on most recent literature survey and we basically highlighted the top-down approach from WSN to recent protocols of routing techniques. We address the challenges faces by WSN and the solution for these constrains. Moreover, we give detailed view for how to apply these techniques to make more efficient protocol using optimization.

The rest of this paper is organized as follows: Section 2 discuss communication architecture. Section 3 comprises the basic routing protocols. Section 4 presents clustering techniques. Section 5 explains the parameters to measure the performance of protocol. Section 6 gives brief introduction about recent proposed protocol by optimization using genetic algorithm. Section 7 concludes this paper.

\section{COMMUNICATION ARCHITECTURE}

As per application specific requirement, number of nodes varies. Each node uses the protocol stack for communication (nodes-nodes, nodes-base-station). It must be reliable and energy efficient. In 2008, Jennifer Yick et.al focused on these layers. Transport layer ensures the reliability, quality of data at the source and the sink, packet-loss recovery (hop-by-hop and end-to-end.), as well as congestion control mechanism. It is application independent but each application suffers from packet loss problem due to packet collision, congestion ,node failure and packet capacity etc. To recover the packet ,hopby-hop technique uses the intermediate node which act as cache that send packet which is lost .This approach is energy efficient as transmission is done with in limited distance. In end-to-end approach, the lost packet will send from its source again which takes more time so effects the QOS. To control congestion, the hop-by-hop technique monitors node by node transmission so that if buffer overflow occurs it will be detected at that time that decreases the retransmission but in end-to-end, the congestion will be detected at last so we need the whole transmission again. Some protocols of this layer are pump slowly fetch quickly, Sensor transmission control protocol and price-oriented reliable transport protocol etc. Network layer handles data routing across the network from the source to the destination so the protocols used for this purpose must be scalable. Motes do not have IP addresses, so IP-based routing protocols cannot be used. The protocols work with in the resource constraints. Geographical routing secure routing and anchor location services are some protocols required for this purpose. Data-link layer handles the data transfer among nodes having same link. It concerned with error detection and correction services. As per wireless network, the MAC protocol is required for effective data transfer. For error detection and correction, the cyclic redundancy check, forward error correction, automatic repeat request etc. are used. Physical layer provides an interface for bit transmission over the physical-communication medium. It minimizes energy consumption and maximizes network lifetime starts from this layer. It communicates with MAC layer for specific tasks such as transmission, reception and modulation to achieve higher performance and resource utilization [1].

\section{ROUTING PROTOCOL}

Motes have some constraints such as restricted transmission range, limited processing, storage capability and energy resources. So to improve the communication after concerning all these issues routing protocols are required. These protocols are responsible for maintaining the routes in the network and reliable multi-hop communication. It is challenging because of unexpected change in node status. There are various traditional routing protocols (such as flooding, Gossiping etc.) but due to energy-constrained nature of network, they have several shortcomings. 
Flooding is a blind technique in which nodes broadcast packets till reaches its destination. It may be possible that duplicate packets circulate in network and sensors will receive redundant packets (Implosion problem). Also when Sensor nodes sense the same geographic areas they gathered overlapping data then flood the data to their common neighbour (Overlapping problem).

In gossiping, a sensor would select randomly one of its neighbours and send the packet to it till sink receive this packet. It tackles the implosion problem but inconvenient when number of nodes increases and also delay occur to reach packet to its destination [2].

The task of these protocols is not only to find the best path (low energy) from source to destination but also to extend network lifetime. Nikolaos A. Pantazis et.al in 2013 [3] focused on these are categorised routing protocols in different scheme as shown in figure 1.

\begin{tabular}{|l|l|l|l|}
\hline \multicolumn{4}{|c|}{ Routing protocol } \\
\hline $\begin{array}{l}\text { Network } \\
\text { Structure }\end{array}$ & $\begin{array}{l}\text { Communication } \\
\text { Model }\end{array}$ & $\begin{array}{l}\text { Topology } \\
\text { Based }\end{array}$ & $\begin{array}{l}\text { Reliable } \\
\text { Routing }\end{array}$ \\
\hline
\end{tabular}

Fig 1: Various category of routing protocols

Network structure deals with the way the nodes are connected like in Hierarchical structure bottom-up approach used for balanced energy structure. It includes flat and Hierarchical routing protocols

Communication model is concerning routing i.e. path for transmission data or packets without focus on structure. It includes query based, coherent and non-coherent based routing protocols

Topology based protocols mainly concern with the topology of network and finding their location on the basis of their neighbour positions. It includes location based and mobility based routing protocols

Reliable routing protocols focus on energy efficient transmission by using multi-path from source to destination. It includes multipath based and QOS based routing protocols.

\section{CLUSTERING PROTOCOLS}

By minimized the number of communication between nodes and reduce energy consumption, the network lifespan improved. Clustering technique is efficient to decrease the communication as it supports various topologies. On the basis of convergence time, these algorithms are classified into constant convergence time algorithm and variable convergence time algorithm. The constant convergence time algorithm completely converges in fixed iterations regardless of the size of population. The variable convergence time algorithm mainly focuses on the time to complete execution. They enable more control of cluster properties instead of constant time [4].

In this technique, the cluster head election is main concern as there are thousands of sensor nodes deployed in unorganized manner. The $\mathrm{CH}$ manages the communication among all its members and manages the data. These protocols are categorized in homogenous, heterogeneous and hybrid based protocols as shown in figure 2 .

\subsection{Homogeneous Sensor Networks:}

Here, all the sensor nodes and base stations are identical in terms of hardware capability and battery power initially. In this network, the static clustering elects cluster heads $(\mathrm{CH})$ only once for the entire lifetime of the network. This results in overhead on cluster heads [5].

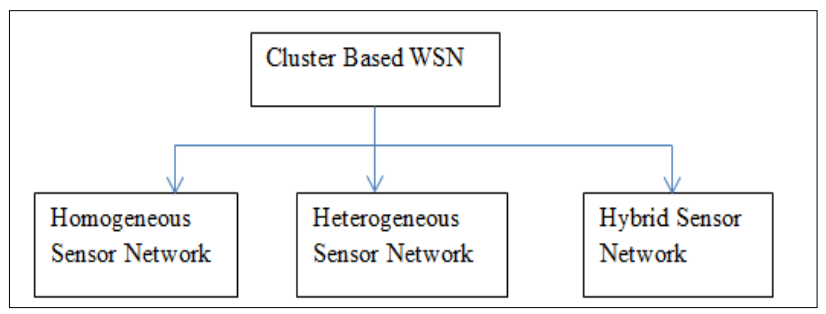

Fig 2: Types of clustering

LEACH is self - organizing clustering protocol which concerns with the equalized energy load distribution among sensor nodes. The whole process is divided into multiple groups and each group is split into two phases that is set-up and steady phase. In setup phase the $\mathrm{CH}$ is elected and in steady phase all the member nodes are in sleep mode to save energy along with the real transmission is done among $\mathrm{CH}$ and the BS. All the sensor nodes are organized into local clusters having the more energy node act as $\mathrm{CH}$ and all other are the members of that local cluster. The transmission is only done via $\mathrm{CH}$ so all the fusion done locally. This prolongs the lifespan of network. The $\mathrm{CH}$ is elected on the basis of no. of times it become $\mathrm{CH}$. Each sensor nodes selects the random value between 0 to 1 if the values is less than threshold then that node become the $\mathrm{CH}$. There are so many variant occur on the basis of different parameters such as selection criteria of $\mathrm{CH}$, multi-hop data transmission, chain based, heterogeneous based mobility based etc. are explained by Sudhanshu tyagi et.al [6]

To make energy efficient homogeneous network the overload on $\mathrm{CH}$ must be bearable and decreases as far as possible. As per this concern the $\mathrm{CH}$ was elected on the basis of residual energy of existing $\mathrm{CH}$, nearest Hop distance of node.

Shio Kumar Singh et.al, 2010 [5] proposed a homogeneous clustering algorithm is to prolong the lifetime of the network by homogeneous distribution of nodes with in the clusters so that overhead on $\mathrm{CH}$ decreases at the receiving and transmitting time. To decrease the overload on $\mathrm{CH}$ it was elected on the basis of residual energy of existing $\mathrm{CH}$, nearest hop distance of node. Here we assume, BS is located far from sensor nodes and having the information of node's location. Each node can send data to BS and having same energy for transmission and reception also having the ' $\mathrm{P}$ ' probability to become $\mathrm{CH}$ at first round. The existing $\mathrm{CH}$ becomes $\mathrm{CH}$ again after $1 / \mathrm{P}$ rounds each node has same energy and consumes equal energy for transmission and reception. While $\mathrm{CH}$ is selected, the nodes must not be dynamic.

\subsection{Heterogeneous Sensor Networks:}

Collection of different types of sensor nodes (two or more than two) having different hardware capabilities and battery power are used. Cluster head is elected on the basis of these criteria. Higher hardware capabilities and more battery power, higher the probability to become $\mathrm{CH}$ node. $\mathrm{CH}$ is used to process and send the information whereas low energy nodes (inexpensive nodes) performed sensing task [7].There are various resources on the basis of heterogeneity is considered 
such as computational heterogeneity, link heterogeneity and energy heterogeneity

Computational heterogeneity includes heterogeneous nodes having powerful microprocessor and large storage space so nodes can perform complex data processing.

Link heterogeneity means the heterogeneous nodes having high-bandwidth and long-distance network transceiver than other nodes. It mainly concern with reliable data transmission.

Energy heterogeneity concerns with power. Here, the heterogonous nodes are line powered or replaceable battery.

To make energy efficient clustering, the efficient organization of nodes into cluster is required. The algorithm for this separates into two different phases (Set-Up, Steady).

To decrease the overhead and make energy efficient clustering, the $\mathrm{CH}$ is selected on the basis of weighted election probability of sensor nodes or the ratio of residual energy of each node to average energy of network [7].

SEP considers the energy as a parameter to elect a cluster head. In this protocol, node elects itself as cluster head independently on the basis of their own initial energy. It increases the stability period which shows the time interval before first dead. They assign the weight to optimal election probability and the weight is the ratio of energy of each node initially to the energy of normal node initially. They assume that the sink has infinite energy and the coordinates are also defined. They split the nodes as advance and normal nodes. The advance nodes mostly become a cluster head. They increases the epoch with increases the energy parameter. According to this protocol, each node knows the energy of whole network. They are aware of heterogeneity of the protocol by assigning the weight. It also deals when the threshold energy is exceeded from nodes. Its performance is better than others because of uniform distribution of advance node in overall network. There are many variants of SEP as well [8].

Femi A. Aderohunmu1 et.al proposed enhanced SEP (SEP-E) considers one other type of node that is intermediate node and its energy lies in between of normal and advance node. This also increases the initial energy of network. The election of $\mathrm{CH}$ is done in same way but threshold value is set to guarantee each node must become a $\mathrm{CH}$. The parameters (no. of alive node, no. of dead node, stability time) to measure the performance of network is considered. This results the more stability and more lifespan by selecting the advance node as the $\mathrm{CH}$ most of times [9].

SEP-E (RCH) proposed by Raju Pal et.al in 2013 is energy efficient and electing redundant $\mathrm{CH}$ to increase the lifetime and reliability of network. The remaining energy and the minimum mean distance from all ordinary nodes to $\mathrm{CH}$ in network are the parameters on the basis of which $\mathrm{CH}$ is elected [10].

Dilip Kumar et.al in 2009 proposed which uses the features of LEACH that is spatial density of network and merge them with node distribution to achieve minimize energy consumption. They use the weighted probability for electing $\mathrm{CH}$, so the energy consumption of each node is in proportion [11].

\subsection{Hybrid sensor network}

In this type of network the sink also contribute to increase the performance of network such as data gathering and assigning the task to node on the basis of their qualities in real time. It includes APTEEN and EEHRP etc. [4].

\section{PERFORMANCE MEASURES}

There are some parameters to measure the performance of the network. Some of these are:

a) Network lifetime: time interval from start of operation in sensor network to the time when first node dead.

b) Number of cluster heads per round: this shows the number of nodes that send data directly to sink after aggregated from all other nodes.

c) Number of alive nodes per round: this reflects the nodes of each type that has not expanded all its energy.

d) Throughput: the rate of data sent from $\mathrm{CH}$ to sink and from node to $\mathrm{CH}$ [11].

\section{OPTIMIZATION}

Clustering is used in much application but gives the NP-hard problems. To solve these problems the optimization tools are required. We survey the recent protocol proposed by applying genetic algorithm for optimization.

Genetic algorithm is the meta-heuristic algorithm based on the evolutionary ideas of natural selection and genetics. It is an optimization technique that selects the best fit node for data transmission. The procedure it uses is:
a) Create initial population,
b) Evaluate fitness,
c) Apply operation selection, crossover and mutation

The fitness value of new population is replaced by previous if it is more than its parent. The algorithm terminates if the energy consumption is below the threshold value [12].

A Balamurugan in 2014 proposed FRP which is used to optimize data transmission efficiently by reduces the energy consumption of nodes. It selects the nodes having minimum hop-count (distance from BS) for transmitting data. For finding the fittest node to route packet the optimization technique (Genetic algorithm) is used. It extend the network lifespan and throughput along with reduces the end-end delay. The whole process is divided in four phases [13].

a) Network Initialization phase: The BS broadcast requesting message (HELLO) to its neighbours that contain the BS address and Hop-Count. After receiving message it saves the Hop-Count value into its memory and increases it by 1 .

b) Data Congregation: After detecting something happening in network, the BS sets its transmission radius and gathers information from these nodes like Hop-Count, residual energy etc.

c) Fitness Calculation: After receiving information, BS selects the next node on the basis of fitness value to transmit the packet. Selection is done on the basis of best fit value calculated by using

$$
\text { Fitness }=\operatorname{dist}(K, L)+\operatorname{dist}(l, B S)
$$

Where, $\mathrm{K}, \mathrm{L}$ are parent nodes, BS is the base station. 
d) Packet Routing: after finding the best node to transmit data, the BS send packet to that node and process repeats from data congregation phase till reaches to the destination node.

During transmission, if any network failure occurs, the network chooses an alternate path. This is done by selecting the other node among the two nodes that were selected in the previous phase. Hence, transmission errors are minimized [13].

B. Baranidharan in 2015 proposed GAECH which forms well-balanced clusters by including the core parameters of a Cluster with in its fitness function, which increases both the stability period, lifespan of the network and reduces the overall energy consumption [14].

Fitness function $\in\left(E_{\text {total }}, S D, C H_{\text {disp }}, E_{\text {Total } \mathrm{CH}}\right)$ eq. (3)

(i) $E_{\text {total }}$ is total energy consumption for single data collection Round):

$$
\begin{gathered}
E_{\text {total }}=E_{\text {intra }}+E_{\text {inter }} \\
\text { Where, } E_{\text {intra }}=\sum_{i=1}^{n} E(i, C H)+E R X+E D A \\
E_{\text {inter }}=\sum_{i=1}^{m} E(i, B S)
\end{gathered}
$$

Where, $(i, \mathrm{CH})$ is energy consumption from $i$ th node to its corresponding $\mathrm{CH}, \mathrm{ERX}$ is the reception energy spent at $\mathrm{CH}, \mathrm{EDA}$ is the data aggregation in $\mathrm{CH}, \mathrm{E}(i, \mathrm{BS})$ is transmission energy from $i$ th $\mathrm{CH}$ to BS, " $n$ " are the cluster member and " $m$ " are cluster head.

(ii) Standard Deviation in energy consumption between

Clusters,

$$
\begin{aligned}
\mu & =\sum_{i=1}^{m} \frac{\operatorname{ECluster}(i)}{m} \\
S D & =\sqrt{\sum_{i=1}^{m}(\mu-\text { ECluster } i)^{\wedge} 2}
\end{aligned}
$$

(iii) $\mathrm{CH}$ dispersion

$$
C H_{\text {disp }}=\min \left\{d\left(\mathrm{CH}_{i}, \mathrm{CH}_{j}\right)\right\}
$$

Where, $\mathrm{CH} i, \mathrm{CH} j \in S$.

" $S$ " is the set of $\mathrm{CH}$ nodes in the current cluster setup network.

(iv) $\mathrm{CH}$ Energy consumption

$$
\begin{gathered}
E_{\text {Total } C H}=\sum_{i=1}^{m} E_{C H i} \\
E_{C H i}=E_{D A}+E_{C H-B S+} E_{R X-m e m b e r}
\end{gathered}
$$

The fitness function $(\mathrm{F})$ of GAECH is represented by

$$
\begin{aligned}
F=W_{1} * E_{\text {Total }}+W_{2} * S D+ \\
W_{3} * \frac{1}{C H_{\text {disp }}}+W_{4} * E_{\text {Total } C H}
\end{aligned}
$$

The constant coefficients $\mathrm{W}_{1}, \mathrm{~W}_{2}, \mathrm{~W}_{3}$ and $\mathrm{W}_{4}$ are the application-dependent weight that indicates the most effective fitness parameter.

\section{CONCLUSION}

WSN is the diversified field from others as it is designed for specific applications. We have surveyed the objective of WSN, issues they faced and how to resolve them by using routing in efficient manner. This paper explains the various clustering techniques to improve the relay, to resolve the computation complexity and to improve the network lifetime. Optimal clustering in the given area is an NP-complete problem. To solve this problem various nature inspired algorithms have been used. We here described the most latest proposed algorithm based on genetic algorithm. These algorithms give better network lifetime as compared with other previous protocols of clustering in WSN. Further, we try to optimize the algorithms by using some other technique.

\section{REFERENCES}

[1] J. Yick, B. Mukherjee, D. Ghosal, "Wireless Sensor Network Survey," Computer Networks, 2008

[2] Shio Kumar Singh , M P Singh , And D K Singh," Routing Protocols In Wireless Sensor Networks - A Survey" International Journal Of Computer Science \& Engineering Survey (Ijcses) Vol.1, No.2, November 2010.

[3] Nikolaos A. Pantazis, Stefanos A. Nikolidakis And Dimitrios D. Vergados, Senior Member, Ieee " Energy-Efficient Routing Protocols In Wireless Sensor Networks: A Survey'Jeee Communications Surveys \& Tutorials, Vol. 15, No. 2, Second Quarter 2013.

[4] Abbasi, Ameer Ahmed, and Mohamed Younis. "A survey on clustering algorithms for wireless sensor networks." Computer communications 30.14: 2826 2841:2007.

[5] Shio Kumar Singh , M P Singh, And D K Singh "Energy Efficient Homogenous Clustering Algorithm For Wireless Sensor Networks" International Journal Of Wireless \& Mobile Networks ( Ijwmn ), Vol.2, No.3, August 2010.

[6] Tyagi, Sudhanshu, and Neeraj Kumar. "A systematic review on clustering and routing techniques based upon LEACH protocol for wireless sensor networks." Journal of Network and Computer Applications 36.22013

[7] Vivekkatiyar, Narottam Chand, surendersoni "Clustering Algorithms For Heterogeneous Wireless Sensor Network: A Survey" International Journal Of Applied Engineering Research, Dindigul Volume 1, No 2, 2010.

[8] Smaragdakis, Georgios, Azer Bestavros, and Ibrahim Matta. SEP: A stable election protocol for clustered heterogeneous wireless sensor networks. Boston University Computer Science Department, 2004.

[9] Aderohunmu, Femi A., and Jeremiah D. Deng. "An enhanced stable election protocol (sep) for clustered heterogeneous wsn." Department of Information Science, University of Otago, New Zealand (2009). 
[10] Pal, Raju, Ritu Sindhu, and Ajay K. Sharma. "SEP-E (RCH): Enhanced Stable Election Protocol Based on Redundant Cluster Head Selection for HWSNs." International Conference on Heterogeneous Networking for Quality, Reliability, Security and Robustness. Springer Berlin Heidelberg, 2013.

[11] Kumar, Dilip, Trilok C. Aseri, and R. B. Patel. "EEHC: Energy efficient heterogeneous clustered scheme for wireless sensor Communications 32.4. 2009
[12] Mukhopadhyay, Deep Malya, et al. "Genetic algorithm: A tutorial review." International Journal of of Grid and Distributed Computing $2.3: 25-32 .: 2009$

[13] A Balamurugan " An Energy Efficient Fitness Based Routing Protocol In Wireless Sensor Networks"Ictact Journal On Communication Technology, Volume: 05, Issue: 01 March 2014.

[14] B. Baranidharan And B. Santhi "Gaech: Genetic Algorithm Based Energy Efficient Clustering Hierarchy In Wireless Sensor Networks" Hindawi Publishing Corporation Journal Of Sensors Volume 2015. 